age was 40 years (IQR = 31-51), 43/142 (30\%) were HIV positive, $38 / 142(27 \%)$ had been diagnosed and treated for syphilis in the past and 11/142 (8\%) presented with symptoms (possible lesions of primary or secondary syphilis). Thirteen $(9 \%, 95 \% \mathrm{CI}=4.4-13.9)$ tested positive for syphilis on the day of presentation and all were treated presumptively. MSM who were symptomatic (genital ulcer or body rash), HIV sero-positive or had a history of previous syphilis were significantly more likely to test positive for syphilis $(\mathrm{OR}=51.88$, 95\%CI3.01-893.14, $\mathrm{p}=0.007)$.

Factors associated with acquiring syphilis amongst MSM presenting as sexual contacts of syphilis were; being HIV seropositive, having a previous history of syphilis, or presenting with symptoms (possible lesions of primary or secondary syphilis).

\section{P044 TOWARDS THE SPREAD OF THE NEW L2-L2B/D-DA HYBRID VARIANT IN MEN WHO HAVE SEX WITH MEN IN FRANCE?}

${ }^{1} \mathrm{~A}$ Touati, ${ }^{1,2} \mathrm{M}$ Carrer, ${ }^{1,2} \mathrm{C}$ Bébéar, ${ }^{1,2} \mathrm{~B}$ de Barbeyrac, ${ }^{1,2} \mathrm{O}$ Peuchant*. ${ }^{1}$ Bordeaux University Hospital, Department of Bacteriology, National Reference Centre for bacterial Sexually Transmitted Infections, Bordeaux, France; '2Univ. Bordeaux, USC EA 3671, Mycoplasmal and chlamydial humans infections, Bordeaux, France

\subsection{6/sextrans-2021-sti.189}

Background Recent European studies reported lymphogranuloma venereum (LGV) cases caused by a recombinant Chlamydia trachomatis strain presenting L2-L2b/D-Da hybrid ompA sequence, which mostly affected high-risk group of men who have sex with men (MSM). Given the potential risk of dissemination of this hybrid variant, it seemed important to take stock of the situation in France.

Methods All LGV-positive anorectal specimens collected in the French National Reference Centre for bacterial Sexually Transmitted Infections (STIs) in 2018 from HIV-positive MSM with anorectal symptoms were selected. All specimens were analysed by sequencing of the ompA gene. The obtained sequences were compared by alignment with currently available chlamydial L genovars-ompA gene sequences.

Results A total of 184 LGV-positive anorectal specimens matched the selection criteria. The mean age of patients was 40.7 years [23-68]. The ompA gene was successfully sequenced for 146/184 specimens. Most specimens had ompA sequences identical to that of C. trachomatis L2 (41.7\%, 61/ $146)$ and L2b strains $(36.3 \%, 53 / 146)$. We also identified the co-circulation of several genovariants: six (4.1\%) L2-L2b/D-Da hybrid variants, two (1.3\%) L2 variants (one specimen with the A997G susbtitution and one with the G868A substitution) and $27(18.4 \%)$ L2b ompA variants (L2bv1 n=12, L2bv2 $n=3$, L2bv3 $n=1$, L2bv6 $n=5$, L2bv8 $n=1$, new L2b variant C340G $n=1$ ). We also found one specimen with L1 ompA genotype.

Conclusion The circulation of the recently described L2-L2b/ D-Da hybrid variant is sparse (4.1\%) in France whereas it represents $16.9 \%$ of LGV cases in Italy and $12.5 \%-16.5 \%$ of LGV cases in Portugal. Patient's clinical characteristics did not differ with regard to the different LGV variants. Further investigations are needed to better understand the transmission dynamics of this hybrid variant.

\section{P045 ASSESSMENT OF NEISSERIA GONORRHEA PREVALENCE AND RESISTANCE IN THE EASTERN MEDITERRANEAN REGION}

I Maatouk*. Nottingham Trent University

10.1136/sextrans-2021-sti.190

Background Neisseria gonorrhea (NG) has been identified as a high-priority public health concern because of a widespread antimicrobial resistance. The objective is to discuss the findings and implications of this emergence in the Eastern Mediterranean Region (EMR).

Methods A review of paper, reports and guidelines from the region about NG was conducted.

Results In 2015, the estimated prevalence of NG in the EMR was $0.7 \%$ for women and $0.6 \%$ for men compared to global estimations of $0.9 \%$ and $0.7 \%$ respectively. Prevalence in 2016 was higher by $0.2 \%$ for both men and women. Concerning antimicrobial resistance, regional countries from the EMR reporting data on gonococcal isolates with resistance to azithromycin, ciprofloxacin and extended-spectrum cephalosporins (ESCs) were 2 in 2009; 0 in 2010 and only 1 from 2011 to 2014 . The reported data show full susceptibility to ESCs and azithromycin and $>90 \%$ resistance to ciprofloxacin. Another report mentioned 1 reporting country in 2015 and 2016 respectively with resistances of $>90 \%$ for ciprofloxacin. Cefixime, Ceftriaxone and azithromycin resistance testing were negative. Empirical articles are very scarce. In Lebanon $(n=53)$, resistance prevalence was $38.3 \%$ to ciprofloxacin and $40.4 \%$ to azithromycin. Two papers from Morocco $(\mathrm{n}=72$ and $\mathrm{n}=135)$ reported ciprofloxacin resistance in $86.8 \%$ and $77.9 \%$ respectively in 2013 and 2018 without ceftriaxone resistance detected. A case report from Egypt was resistant to ceftriaxone and ciprofloxacin but not to azithromycin.

Discussion It is consistently clear that the EMR has the lowest number of reporting countries compared with all other regions. A call for exploration of NG epidemiology and resistance is recommended through a regional action plan to control and minimize the impact of this public health issue. The promising diagnostic technologies in NG would be beneficial for both the general and the key populations in the absence of financial support to cover current tests.

\section{P047 SEXUAL HEALTH AMONG HIV-NEGATIVE GAY AND BISEXUAL MEN IN LEBANON: A COMPARISON BETWEEN NATIVE AND IMMIGRANT/REFUGEE COMMUNITIES}

I Maatouk*, M Assi, R Jaspal. Nottingham Trent University

10.1136/sextrans-2021-sti.191

Background Unlike native gay and bisexual male (GBM), immigrants/refugees GBM have an added vulnerability to poor sexual health due to their stigmatized sexual minority status. The objective of this cross-sectional study was to compare sexual health variables in a sample of native-born and immigrants/refugees GBM in Lebanon. This study also aimed to identify the correlates of sexually transmitted infections (STIs) knowledge. 